\title{
Effects of planting dates on the growth and grain yield of two indigenous varieties of pearl millet (Pennisetum glaucum (L.) R.Br.) in a forest-savanna transition zone of Edo State, Nigeria
}

\author{
Sunday Ebonka NWAJEI ${ }^{1,2}$, Anthony Uhunomwan OMOREGIE ${ }^{1}$, Felix Omonkeke OGEDEGBE ${ }^{1}$
}

Received September 23, 2018; accepted October 10, 2019

Delo je prispelo 23. septembra 2018, sprejeto 10. oktobra 2019.

Effects of planting dates on the growth and grain yield of two indigenous varieties of pearl millet (Pennisetum glaucum (L.) R.Br.) in a forest-savanna transition zone of Edo State, Nigeria

Abstract: Effects of planting dates on the growth and grain yield of two indigenous varieties of pearl millet was carried out at the Teaching and Research Farm, Ambrose Alli University, Ekpoma. The experiment was a $2 \times 5$ factorial scheme fitted into a randomized complete block design with the two varieties of pearl millet ('Gero Bida' and 'Gero Badeggi') and five planting dates (April, May, June, July and August) replicated three times. The results obtained showed that delays in planting date significantly reduced growth in pearl millet examined. Similar pattern was observed on days to $50 \%$ flowering and grain maturity. Improved growth with early sowing could have allowed increased availability of assimilates that later supported reproductive growth. These assimilates were remobilized under unfavourable climatic condition in the late cropping season to the reproductive structures. 'Gero Badeggi' sown in April, had significantly, the highest growth in the measured characters than 'Gero Bida' sown in August and other dates. 'Gero Badeggi' planted in May had the highest total grain yield $\left(9.33 \mathrm{t} \mathrm{ha}^{-1}\right)$ while 'Gero Bida' planted in July had the smallest $\left(4.27 \mathrm{t} \mathrm{ha}^{-1}\right)$. Therefore, 'Gero Baddegi' planted in May is a better variety for pearl millet grain production in Ekpoma.

Key words: pearl millet; Pennisetum glaucum; planting date; growth; yield; forest savanna; indigenous variety
Učinki datuma setve na rast in pridelek zrnja dveh avtohtonih sort bisernega prosa (Pennisetum glaucum (L.) R.Br.) $\mathrm{v}$ prehodnem območju gozdnate savane, država Edo, Nigeria

Izvleček: Učinki datuma setve na rast in pridelek zrnja dveh avtohtonih sort bisernega prosa so bili preučevani na Research Farm, Ambrose Alli University, Ekpoma. Poskus je bil narejen kot $2 \times 5$ faktorski naključni bločni poskus z dvema sortama bisernega prosa ('Gero Bida' in 'Gero Badeggi'), petimi datumi setve (april, maj, junij, julij in avgust), s tremi ponovitvami. Rezultati so pokazali, da je zakasnitev v datumu setve značilno zmanjšala rast obeh sort. Podoben učinek je bil na dneve do $50 \%$ cvetenja in zrelost zrnja. Izboljšana rast po zgodnji setvi je povečala količino asimilatov, kar je potem povečalo reproduktivno rast. Ti asimilati so se remobilizirali kasneje $\mathrm{v}$ neugodnih klimatskih razmerah proti koncu rastne sezone in povečali tvorbo reproduktivnih struktur. Sorta Gero Badeggi, sejana aprila je imela značilno največjo rast med merjenimi parametri, v primerjavi s sorto Gero Bida, posejano avgusta in v drugih terminih. 'Gero Badeggi', posejana maja je imela največji celokupni pridelek zrnja (9,33 t ha $\left.{ }^{-1}\right)$, 'Gero Bida’posejana julija pa je imela najmanjšega $\left(4,27 \mathrm{t} \mathrm{ha}^{-1}\right)$. Zaradi naštetega je 'Gero Baddegi', posejana maja, primernejša sorta za pridelovanje zrnja bisernega prosa v Ekpomi.

Ključne besede: biserno proso; Pennisetum glaucum; datum setve; rast; pridelek; gozdnata savana; avtohtona sorta

1 Ambrose Alli University, Department of Crop Science, Ekpoma, Edo State, Nigeria

2 Corresponding author, e-mail: sodje4real10@gmail.com 


\section{INTRODUCTION}

Pearl millet (Pennisetum glaucum (L.) R.Br.) is a drought tolerant cereal typically grown as grain crop in semi-arid and dry areas of the world (Leila, 2008). The crop can potentially be planted as a double crop in northern areas of Nigeria. This crop produces high quality grains than any other cereals under extreme conditions like unfertile soil, intense heat and prolong drought (Amanullah et al., 2015).

The pearl millet is a cereal crop grown mostly for its grain and fodder. It is a robust, tufted, tillering annual grass, up to $300 \mathrm{~cm}$ high (Khan et al., 2003). Pearl millet is locally known as "Gero" and an important grain and fodder crop for savanna areas of Nigeria (Uzoma et al., 2010). Pearl millet can grow in areas with annual rainfall of $250-900 \mathrm{~mm}$ with optimum temperature of $30-35^{\circ} \mathrm{C}$ (Raemaeker, 2001). The ideal soil $\mathrm{pH}$ range is $5.5-7.0$, but it can grow in soil with $\mathrm{pH}$ as low as 4 and as high as 8.3 (Fahmy et al., 2010).

The world's area planted with pearl millet is about sixty five million hectares, with the major part in India and Africa (Raemaeker, 2001). World production of pearl millet stands at around 30 million tonnes annually with $97 \%$ of this figure produced in developing countries (Gabatshele et al., 2014). In Africa, the total production of this crop is about 14 million tonnes annually, with the highest percentage produced in West Africa. In West Africa, about 13 million tonnes is produced with Nigeria, Niger, Burkina-Faso, Chad, Mali and Senegal contributing about $94 \%$ of this annual figure. In Nigeria, the annual production record stands at about 5 million tonnes (Amodu et al., 2005).

The pearl millet is of great importance in the arid and semi-arid tropics, where it is the staple food for millions of people. Today, this crop covers the food needs of more than 500 million people in the world. It is the second most important only to sorghum, as a staple food in the savanna areas of Nigeria (Khan et al., 2003). In Nigeria, pearl millet grains are used primarily for human consumption, feeds for poultry and source of beer for brewing industries.

Planting date plays an important role on growth, development and yield of cereal crops. To pearl millet, it ensures sufficient vegetative growth and grain development for optimum harvesting (Amodu et al., 2005). In Northern Guinea Savanna zone of Nigeria, sowing dates affect pearl millet production, which must be optimal in order to successfully compete with weeds, absorb nutrient and moisture for good growth and development (Shinggu and Gani, 2012). Planting dates must be chosen within a window of opportunity defined by cropping system and available soil moisture.
It also dictates to a large degree how tall the plants will get, and the potential impact of certain insects and diseases on the crop. It also determine the size of the root system, which in turn determines how much stored water the plant can utilize. It can be manipulated to counter the adverse effects of environmental stress (Uzoma et al., 2010).

Planting date can have dramatic effect on crop yield. In fact, proper planting time is important for maximizing pearl millet grain yield (Leila, 2008). In fact, Lawn et al. (1993) have argued that the differences in development of cereals sown at different times may be explained by considering an optimum temperature above which rate of development decreases. Planting time recommendation for pearl millet grain yields is commonly made based on calendar year and soil temperature (Andrews et al., 1998).

Previous study on pearl millet in Edo State focused on the evaluation of some varieties in this zone. 'Gero Bida' and 'Gero Badeggi' varieties performed best in both growth and yield characters (Omoregie \& Nwajei, 2015). Hence, to obtain better growth and grain yield in this zone, it is necessary to determine the appropriate planting date since pearl millet is affected by environmental conditions, particularly photoperiod.

Besides, with dwindling revenue from petroleum and its products and the government policy to diversify the economy, there is need to revamp the agricultural sector, particularly, the cultivation of arable crops beyond their traditional agro-ecological zones to provide sufficient food and income for the ever increasing population.

The research objective was to determine the appropriate planting time on the growth and grain yield of two indigenous varieties of pearl millet in a forestsavanna transition zone of Edo

\section{MATERIALS AND METHODS}

\subsection{EXPERIMENTAL SITE}

A field experiment was carried out during 2016 cropping season in Ekpoma. Ekpoma is located in a forest-savannah transition zone of Edo State, Nigeria. The area is located between Latitude $6^{\circ} 45^{\prime}$ North and Longitude $6^{\circ} 08^{\prime}$ East and has a mean air temperature of $29{ }^{\circ} \mathrm{C}$, relative humidity of $70 \%$, sunshine of about 5 - 7 hours/day and a mean annual rainfall of 1200 - $1500 \mathrm{~mm}$ as established by Ighalo and Remison (2010). 
Table 1: Description of the study varieties used in the experiment

\begin{tabular}{llllllll}
\hline & Name of variety & \multicolumn{2}{l}{ Morphological traits } & & & & \\
\cline { 3 - 8 } NO & & $\begin{array}{l}\text { Height } \\
(\mathrm{cm})\end{array}$ & Tiller & $\begin{array}{l}\text { Synchronous } \\
\text { tillers }\end{array}$ & Head type & Seed colour & $\begin{array}{l}\text { Maturity } \\
\text { days }\end{array}$ \\
\hline 1 & Gero Bida & $75-250$ & $2-9$ & $3-15$ & Terminal-panicle & Brown & $75-90$ \\
2 & Gero Badeggi & $80-300$ & $4-12$ & $2-10$ & Terminal-panicle & Brown & $80-95$ \\
\hline
\end{tabular}

Source: Omoregie and Nwajei (2015)

\subsection{MATERIALS USED}

'Gero Bida' and 'Gero Badeggi' varieties of pearl millet used were obtained from National Cereals Research Institute, Badeggi, Niger State, Nigeria. The description of the pearl millet varieties are given below;

\subsection{SOIL ANALYSIS}

Prior to sowing, soil samples were collected at a depth of $0-15 \mathrm{~cm}$ with an auger. The soil samples were bulked, air dried and sieved with a $2 \mathrm{~mm}$ mesh sieve for the determination of the physical and chemical properties using the method described by Anderson and Ingram (1993).

\subsection{PLANTING DATES AND EXPERIMENTAL DE- SIGN}

The two varieties were planted at five dates namely: April $10^{\text {th }}$, May $10^{\text {th }}$, June $10^{\text {th }}$, July $10^{\text {th }}$ and August $10^{\text {th }}$ to give ten treatment combinations. The experiment was a $2 \times$ 5 factorial scheme fitted into a randomized complete block design with three replicates to give a total of thirty plots.

\subsection{LAND PREPARATION AND PLANTING}

The land was manually prepared and plots demarcated according to treatment. A pinch of seeds of each of the two varieties were sown on minimally prepared beds, monthly, starting from $10^{\text {th }}$ April to $10^{\text {th }}$ August 2016 and later thinned to one plant per stand at one week after planting (WAP). The gross plot size was $1.25 \times 3 \mathrm{~m}$. The planting spacing used was $25 \times 75 \mathrm{~cm}$ intra- and inter rows, equivalent to 53,333 plants/ ha with a spacing of $1 \mathrm{~m}$ within plots and between replicates.

\subsection{WEED CONTROL}

Weeding was conducted manually at 3 and 7 followed by supplementary weeding at 12 WAP.

\subsection{SAMPLING AND DATA COLLECTION}

\subsubsection{Growth parameters}

A sample of four plants per plot was taken at two middle rows at two weeks intervals until crops were harvested to measure the following parameters. Plant height $(\mathrm{cm})$ was taken as the height of the main culm from ground level to the tip of the plant with a measuring tape (Remison and Eifediyi, 2014) while the number of leaves per plant was determined by visual counting. The stem girth $(\mathrm{cm})$ was measured by using a digital vernier caliper $(150 \mathrm{~mm} \times 0.01 \mathrm{~mm}$ IP54, BEAPO Hardware Industrial Co, LTD, Changsha, Hundan, China) at $2 \mathrm{~cm}$ above ground level. Total leaf area per plant $\left(\mathrm{cm}^{2}\right)$ was estimated from leaf length multiplied by the widest width, multiplied by a constant 0.75 (Remison and Eifediyi, 2014) and there after multiplied by total number of leaves/plant while number of tillers and synchronous tillers (branch) /plant (Obeng et al., 2012; Omoregie and Nwajei, 2015) were counted visually.

\subsubsection{Flowering and maturity traits}

Days to $50 \%$ flowering was taken as the number of days from planting to the time when $50 \%$ of the plants population produced flowers while days to $50 \%$ maturity was recorded from the date of planting to the day $50 \%$ of the plant populations of the pearl millet had matured panicles.

\subsubsection{Grain yield and grain yield components}

Number of terminal panicles, tiller panicles and synchronous tillers (branch) panicles/plant were visually counted. Measuring tape was used to take the length of the terminal panicles. A sensitive scale was used to take the mass of 1000 seeds while a weighing balance was used to measure the grain yield from the terminal, tiller, synchronous tiller panicles and the husk 
mass. The total grain yield was determined by the addition of the grain yield from the panicles of the terminal, tillers and synchronous tillers (branch) (Obeng et al., 2012; Omoregie and Nwajei, 2015).

\subsection{DATA ANALYSIS}

All growth, flowering and maturity characters and grain yield data obtained were computed using the Statistical Analysis System (SAS) package version 9.0 (2002) and the means separated using Duncan's Multiple Range Test (DMRT) at 0.05 level of probability.

\section{RESULTS}

\subsection{SOIL PROPERTIES OF THE EXPERIMENTAL SITE}

The results of the soil properties show that the textural class was sandy loam, slightly acidic (5.80) with marginal organic carbon $\left(8.40 \mathrm{~g} \mathrm{~kg}^{-1}\right)$, low nitrogen content $\left(0.43 \mathrm{~g} \mathrm{~kg}^{-1}\right)$, moderate available phosphorus (13.05 $\mathrm{mg} \mathrm{kg}^{-1}$ ) and exchangeable bases (Table 2).

\subsection{PLANT HEIGHT}

The effects of planting date on plant height of two varieties of millet are shown in Table 3. The height increased significantly with time until 10 WAP were no further increase. At $14 \mathrm{WAP}$, plant height ranged significantly from $166.44-203.17 \mathrm{~cm}$ in 'Gero bida' and $181.00-233.53 \mathrm{~cm}$ in 'Gero Badeggi'. In all, 'Gero Badeggi' developed taller stems with an average of $208.97 \mathrm{~cm}$ than 'Gero Bida' $(183.33 \mathrm{~cm})$. April sown crops had the tallest stems while those of August had the shortest.

\subsection{NUMBER OF LEAVES/PLANT}

Number of leaves/plant of the two varieties of pearl millet increased from 2 WAP up to 10 WAP and thereafter decreased significantly up to 14 WAP (Table 4). At 14 WAP, the mean varied from $7.00-10.42$ in 'Gero Bida' and 8.42 - 12.33 in 'Gero Badeggi'. On the whole, 'Gero Badeggi' had higher number of leaves/ plant than 'Gero Bida.' The mean number of leaves per plant for 'Gero Badeggi' was 11.12 while that of 'Gero Bida’ was 9.35. Crops planted in April produced the
Table 2: Main soil properties of the experimental site prior to planting

\begin{tabular}{|c|c|}
\hline Parameters & Values \\
\hline Sand $\left(\mathrm{g} \mathrm{kg}^{-1}\right)$ & 830 \\
\hline Silt $\left(\mathrm{g} \mathrm{kg}^{-1}\right)$ & 60 \\
\hline Clay $\left(\mathrm{g} \mathrm{kg}^{-1}\right)$ & 110 \\
\hline Textural class & Sandy loam \\
\hline $\mathrm{pH}\left(\mathrm{H}_{2} 01: 1\right)$ & 5.80 \\
\hline Organic carbon $\left(\mathrm{g} \mathrm{kg}^{-1}\right)$ & 8.40 \\
\hline Total nitrogen $\left(\mathrm{g} \mathrm{kg}^{-1}\right)$ & 0.43 \\
\hline Available phosphorus (mg kg-1) & 13.05 \\
\hline Exchangeable calcium $\left(\mathrm{cmol} \mathrm{kg}^{-1}\right)$ & 1.04 \\
\hline Exchangeable magnesium ( $\mathrm{cmol} \mathrm{kg}^{-1}$ ) & 0.45 \\
\hline Exchangeable potassium $\left(\mathrm{cmol} \mathrm{kg}^{-1}\right)$ & 1.03 \\
\hline Exchangeable sodium $\left(\mathrm{cmol} \mathrm{kg}^{-1}\right)$ & 0.07 \\
\hline \multicolumn{2}{|l|}{ Total exchangeable bases $\left(\mathrm{cmol} \mathrm{kg}^{-1}\right)$} \\
\hline$\left[\mathrm{Ca}^{2+}+\mathrm{Mg}^{2+}+\mathrm{K}^{+}+\mathrm{Na}^{+}\right]$ & 2.59 \\
\hline \multicolumn{2}{|l|}{ Total exchangeable acidity $\left(\mathrm{cmol} \mathrm{kg}^{-1}\right)$} \\
\hline$\left[\mathrm{Al}^{3+}+\mathrm{H}^{+}\right]$ & 0.12 \\
\hline Effective cation exchange capacity $\left(\mathrm{cmol} \mathrm{kg}^{-1}\right)$ & 2.71 \\
\hline Base saturation \% & 95.57 \\
\hline
\end{tabular}

highest while those of August had the least number of leaves.

\subsection{NUMBER OF SYNCHRONOUS TILLERS (BRANCHES)/PLANT}

Results on number of branches per plant of two millet varieties revealed that branch production commenced at 6 WAP for both crops planted in April and May, while other planting dates started at 8 WAP (Table 5). With the exception of May planted crops, which peak was at 12 WAP other crops planted at other dates reached their maximum branch production at $10 \mathrm{WAP}$. At 14 WAP, the mean number of branches/plant ranged from 1.67- 5.42 and 1.67 - 4.00 in 'Gero Bida' and 'Gero Badeggi', respectively. Crops planted in May had the highest, while those of July had the least number of branches/plant. In all, both varieties gave similar number of synchronous tillers, that is, approximately 3 . 
Table 3: Effect of planting date on the plant height $(\mathrm{cm})$ of two varieties of pearl millet at Ekpoma

\begin{tabular}{|c|c|c|c|c|c|c|c|c|}
\hline \multirow[t]{2}{*}{ Crop variety } & \multirow[t]{2}{*}{ Planting date } & \multicolumn{7}{|c|}{ Weeks after planting } \\
\hline & & 2 & 4 & 6 & 8 & 10 & 12 & 14 \\
\hline \multirow[t]{6}{*}{ Gero Bida } & April & $4.46^{\mathrm{a}}$ & $17.88^{\mathrm{a}}$ & $81.00^{\mathrm{a}}$ & $174.42^{\mathrm{b}}$ & $203.17^{\mathrm{bcd}}$ & $203.17^{\mathrm{bcd}}$ & $203.17^{\mathrm{bcd}}$ \\
\hline & May & $3.82^{\mathrm{abcd}}$ & $12.46^{\mathrm{b}}$ & $55.92^{\mathrm{b}}$ & $177.08^{\mathrm{b}}$ & $193.50^{\text {cde }}$ & $193.50^{\text {cde }}$ & $193.50^{\text {cde }}$ \\
\hline & June & $3.68^{\mathrm{abcd}}$ & $11.17^{\mathrm{b}}$ & $38.08^{\mathrm{cd}}$ & $157.01^{\mathrm{b}}$ & $177.58^{\mathrm{de}}$ & $177.58^{\mathrm{de}}$ & $177.58^{\mathrm{de}}$ \\
\hline & July & $3.17^{\mathrm{cd}}$ & $10.25^{\mathrm{bc}}$ & $44.17 b^{c}$ & $135.92^{\mathrm{c}}$ & $175.99^{\mathrm{de}}$ & $175.99^{\mathrm{de}}$ & $175.99^{\mathrm{de}}$ \\
\hline & August & $3.83^{\mathrm{abcd}}$ & $7.63^{\mathrm{c}}$ & $24.67^{\mathrm{de}}$ & $111.00^{\mathrm{d}}$ & $166.44^{\mathrm{e}}$ & $166.44^{\mathrm{e}}$ & $166.44^{\mathrm{e}}$ \\
\hline & Mean & 3.79 & 11.88 & 48.80 & 151.09 & 183.33 & 183.33 & 183.33 \\
\hline \multirow[t]{10}{*}{ Gero Badeggi } & April & $4.29^{\mathrm{ab}}$ & $18.21^{\mathrm{a}}$ & $91.69^{\mathrm{a}}$ & $211.50^{\mathrm{a}}$ & $233.53^{\mathrm{a}}$ & $233.53^{\mathrm{a}}$ & $233.53^{\mathrm{a}}$ \\
\hline & May & $3.99^{\mathrm{abc}}$ & $12.42^{\mathrm{b}}$ & $42.25^{c}$ & $164.92^{\mathrm{b}}$ & $226.83^{\mathrm{ab}}$ & $226.83^{\mathrm{ab}}$ & $226.83^{\mathrm{ab}}$ \\
\hline & June & $3.47^{\mathrm{bcd}}$ & $13.25^{\mathrm{b}}$ & $36.50^{\mathrm{cd}}$ & $156.92^{\mathrm{b}}$ & $213.67^{\mathrm{abc}}$ & $213.67^{\mathrm{abc}}$ & $213.67^{\mathrm{abc}}$ \\
\hline & July & $3.08^{\mathrm{d}}$ & $10.67^{b c}$ & $27.00^{\mathrm{de}}$ & $131.58^{c}$ & $189.75^{\text {cde }}$ & $189.75^{\text {cde }}$ & $189.75^{\text {cde }}$ \\
\hline & August & $3.46^{\mathrm{bcd}}$ & $7.71^{\mathrm{c}}$ & $17.42^{\mathrm{e}}$ & $117.58^{\mathrm{cd}}$ & $181.00^{\mathrm{de}}$ & $181.00^{\mathrm{de}}$ & $181.00^{\mathrm{de}}$ \\
\hline & Mean & 3.66 & 12.45 & 42.92 & 156.50 & 208.97 & 208.97 & 208.97 \\
\hline & SL & & & & & & & \\
\hline & Planting date & * & * & * & * & * & * & * \\
\hline & Variety & ns & ns & * & * & * & * & * \\
\hline & $\begin{array}{l}\text { Interaction } \\
(\mathrm{V} \times \mathrm{D})\end{array}$ & * & * & * & * & * & * & * \\
\hline
\end{tabular}

Values followed by the same superscript(s) indicated in columns are not significantly different at the $5 \%$ probability level when using Duncan multiple range test. SL- level of significance; ${ }^{*}$ significant at the 0.05 probability level; ns: values not significant; V-variety; D- planting date

\subsection{TOTAL LEAF AREA/PLANT}

Total leaf area per plant increased steadily with age up to 10 WAP and thereafter decreased until 14 WAP (Table 6). At 14 WAP, the mean varied from 5345.99$9952.05 \mathrm{~cm}^{2}$ and $6187.02-13060.09 \mathrm{~cm}^{2}$ in 'Gero Bida' and 'Gero Badeggi', respectively. In all, 'Gero Badeggi' significantly $(\mathrm{p}<0.05)$ had larger total leaf area $(10,431.13$ $\left.\mathrm{cm}^{2}\right)$ than 'Gero Bida' $\left(8,415.20 \mathrm{~cm}^{2}\right)$. Total leaf area decreased with time of planting from April to August. Crops planted in April significantly gave the largest total leaf area/plant while those of August had the least.

\subsection{NUMBER OF TILLERS/PLANT}

Tillering in 'Gero Badeggi' was significantly higher than in 'Gero Bida' (Table 7). An average of 3 tillers/plant was obtained for 'Gero Badeggi' while that of 'Gero Bida' was 2 tillers/ plant. With the exception of crops planted in April, which tillering started at 2 WAP, May to August plants commenced tillering at 4 WAP. Number of tillers increased steadily with time until 8 WAP for both varieties and thereafter decreased till 14 WAP. At 14 WAP, the number of tillers significantly ranged from $1.08-3.25$ in 'Gero Bida' and 2.58 - 3.83 in 'Gero Badeggi'. April planted crops significantly had the highest number of tillers/ plant while those of August had the least.

\subsection{STEM GIRTH}

The maximum stem girths produced by both varieties were comparable and had no significant difference (Table 8). The stem girth increased from $2 \mathrm{WAP}$ up to 10 and 12 WAP for both varieties. At $14 \mathrm{WAP}$, the stem girth varied from $1.68-2.19 \mathrm{~cm}$ in 'Gero Bida' and $1.86-2.54$ in 'Gero Badeggi'. Although 'Gero Badeggi' significantly produced bigger stems than 'Gero Bida' both had similar girth sizes; approximately $2 \mathrm{~cm}$. April planted crops significantly had the biggest girth than other planting dates. The smallest girth was obtained in August.

\subsection{DAYS TO50 \% FLOWERING}

Plants sown in April needed higher number of days to flowering while those of May needed lower 
Table 4: Effect of planting date on the plant height $(\mathrm{cm})$ of two varieties of pearl millet at Ekpoma

\begin{tabular}{|c|c|c|c|c|c|c|c|c|}
\hline \multirow[t]{2}{*}{ Crop variety } & \multirow[t]{2}{*}{ Planting date } & \multicolumn{7}{|c|}{ Weeks after planting } \\
\hline & & 2 & 4 & 6 & 8 & 10 & 12 & 14 \\
\hline \multirow[t]{6}{*}{ Gero Bida } & April & $6.42^{\mathrm{a}}$ & $8.08^{\mathrm{a}}$ & $11.53^{\mathrm{ab}}$ & $12.18^{\mathrm{c}}$ & $11.75^{\mathrm{bcd}}$ & $11.00^{\mathrm{cd}}$ & $10.25^{\mathrm{bc}}$ \\
\hline & May & $5.65^{\mathrm{b}}$ & $7.83^{\mathrm{ab}}$ & $10.33^{\mathrm{bc}}$ & $11.42^{\text {cde }}$ & $11.42^{\mathrm{cd}}$ & $9.67^{\mathrm{de}}$ & $9.58^{\mathrm{cd}}$ \\
\hline & June & $4.82^{\mathrm{c}}$ & $7.25^{\mathrm{abcd}}$ & $9.75^{\mathrm{cde}}$ & $11.92^{\mathrm{cd}}$ & $11.42^{\mathrm{cd}}$ & $9.67^{\mathrm{de}}$ & $9.67^{\mathrm{cd}}$ \\
\hline & July & $3.83^{\mathrm{d}}$ & $7.92^{\mathrm{ab}}$ & $9.58^{\text {cde }}$ & $10.75^{\mathrm{e}}$ & $10.92^{\mathrm{d}}$ & $10.50^{\mathrm{cd}}$ & $10.42^{\mathrm{bc}}$ \\
\hline & August & $3.67^{\mathrm{d}}$ & $6.46^{\mathrm{d}}$ & $8.33^{\mathrm{e}}$ & $9.42^{\mathrm{f}}$ & $9.25^{\mathrm{e}}$ & $8.08^{\mathrm{e}}$ & $7.00^{\mathrm{e}}$ \\
\hline & Mean & 4.88 & 7.51 & 9.90 & 11.14 & 10.95 & 9.78 & 9.35 \\
\hline \multirow[t]{10}{*}{ Gero Badeggi } & April & $6.67^{\mathrm{a}}$ & $8.08^{\mathrm{a}}$ & $12.33^{\mathrm{a}}$ & $13.58^{\mathrm{a}}$ & $13.58^{\mathrm{a}}$ & $12.90^{\mathrm{a}}$ & $12.17^{\mathrm{a}}$ \\
\hline & May & $6.17^{\mathrm{ab}}$ & $7.75^{\mathrm{abc}}$ & $10.00^{\mathrm{cd}}$ & $13.08^{\mathrm{ab}}$ & $13.08^{\mathrm{ab}}$ & $12.42^{\mathrm{ab}}$ & $12.33^{\mathrm{a}}$ \\
\hline & June & $4.83^{c}$ & $6.75^{\mathrm{cd}}$ & $9.33^{\text {cde }}$ & $12.33^{\mathrm{bc}}$ & $12.33^{\mathrm{abc}}$ & $11.58^{\mathrm{abc}}$ & $11.50^{\mathrm{ab}}$ \\
\hline & July & $3.83^{\mathrm{d}}$ & $6.58^{\mathrm{d}}$ & $8.83^{\mathrm{de}}$ & $11.58^{\text {cde }}$ & $11.75^{\mathrm{bcd}}$ & $11.25^{\mathrm{bc}}$ & $11.17^{\mathrm{ab}}$ \\
\hline & August & $3.83^{\mathrm{d}}$ & $7.00^{\mathrm{bcd}}$ & $8.33^{\mathrm{e}}$ & $11.17^{\mathrm{de}}$ & $11.19^{\mathrm{cd}}$ & $8.50^{\mathrm{e}}$ & $8.42^{\mathrm{d}}$ \\
\hline & Mean & 5.07 & 7.24 & 9.76 & 12.35 & 12.39 & 11.33 & 11.12 \\
\hline & SL & & & & & & & \\
\hline & Planting date & * & * & * & * & * & * & * \\
\hline & Variety & ns & $\mathrm{ns}$ & $\mathrm{ns}$ & * & * & * & * \\
\hline & $\begin{array}{l}\text { Interaction } \\
(\mathrm{V} \times \mathrm{D})\end{array}$ & * & * & * & * & * & * & * \\
\hline
\end{tabular}

Values followed by the same superscript(s) indicated in columns are not significantly different at the $5 \%$ probability level when using Duncan multiple range test. SL- level of significance; ${ }^{*}$ significant at the 0.05 probability level; ns: values not significant; V-variety; D- planting date

Table 5: Effect of planting date on number of synchronous tillers (branches)/plant of two varieties of pearl millet at Ekpoma

\begin{tabular}{|c|c|c|c|c|c|c|c|c|}
\hline \multirow[t]{2}{*}{ Crop variety } & \multirow[t]{2}{*}{ Planting date } & \multicolumn{7}{|c|}{ Weeks after planting } \\
\hline & & 2 & 4 & 6 & 8 & 10 & 12 & 14 \\
\hline \multirow[t]{6}{*}{ Gero Bida } & April & 0.00 & 0.00 & $2.00^{\mathrm{a}}$ & $3.17^{\mathrm{ns}}$ & $3.78^{\mathrm{ns}}$ & $3.67^{\mathrm{b}}$ & $2.92^{\mathrm{bc}}$ \\
\hline & May & 0.00 & 0.00 & $1.00^{\mathrm{b}}$ & 3.11 & 3.00 & $5.67^{a}$ & $5.42^{\mathrm{a}}$ \\
\hline & June & 0.00 & 0.00 & 0.00 & 2.00 & 2.78 & $2.83^{\mathrm{b}}$ & $2.33^{\mathrm{bc}}$ \\
\hline & July & 0.00 & 0.00 & 0.00 & 1.33 & 2.50 & $1.83^{\mathrm{b}}$ & $1.67^{c}$ \\
\hline & August & 0.00 & 0.00 & 0.00 & 2.50 & 2.67 & $2.67^{\mathrm{b}}$ & $2.08^{c}$ \\
\hline & Mean & 0.00 & 0.00 & 1.50 & 2.42 & 2.95 & 3.33 & 2.88 \\
\hline \multirow[t]{10}{*}{ Gero Badeggi } & April & 0.00 & 0.00 & $1.33^{\mathrm{b}}$ & 2.08 & 2.94 & $2.83^{\mathrm{b}}$ & $2.33^{\mathrm{bc}}$ \\
\hline & May & 0.00 & 0.00 & $1.00^{\mathrm{b}}$ & 2.00 & 2.74 & $5.53^{\mathrm{a}}$ & $4.00^{\mathrm{ab}}$ \\
\hline & June & 0.00 & 0.00 & 000 & 2.00 & 2.67 & $1.93^{\mathrm{b}}$ & $1.67^{\mathrm{c}}$ \\
\hline & July & 0.00 & 0.00 & 0.00 & 2.33 & 3.50 & 2.42 & $2.17^{\mathrm{c}}$ \\
\hline & August & 0.00 & 0.00 & 0.00 & 1.83 & 2.58 & $2.50^{\mathrm{b}}$ & $2.17^{c}$ \\
\hline & Mean & 0.00 & 0.00 & 1.17 & 2.05 & 2.89 & 3.04 & 2.47 \\
\hline & SL & & & & & & & \\
\hline & Planting date & - & - & * & ns & ns & * & * \\
\hline & Variety & - & - & ns & ns & ns & ns & ns \\
\hline & $\begin{array}{l}\text { Interaction } \\
(\mathrm{V} \times \mathrm{D})\end{array}$ & - & - & * & ns & $\mathrm{ns}$ & * & * \\
\hline
\end{tabular}

Values followed by the same superscript(s) indicated in columns are not significantly different at the $5 \%$ probability level when using Duncan multiple range test. SL- level of significance; ${ }^{*}$ significant at the 0.05 probability level; ns: values not significant; V-variety; D- planting date 
Table 6: Effect of planting date on the total leaf area ( $\mathrm{cm}^{2} /$ plant) of two varieties of pearl millet at Ekpoma

\begin{tabular}{|c|c|c|c|c|c|c|c|c|}
\hline \multirow[t]{2}{*}{ Crop variety } & \multirow[t]{2}{*}{ Planting date } & \multicolumn{7}{|c|}{ Weeks after planting } \\
\hline & & 2 & 4 & 6 & 8 & 10 & 12 & 14 \\
\hline \multirow[t]{6}{*}{ Gero Bida } & April & $166.98^{\mathrm{b}}$ & $2095.46^{\mathrm{ab}}$ & $7029.44^{\mathrm{b}}$ & $10011.84^{\mathrm{bcd}}$ & $11412.57^{b c}$ & $10682.31^{b c}$ & $9952.05^{b c}$ \\
\hline & May & $72.91^{\mathrm{c}}$ & $1721.27^{\mathrm{bc}}$ & $5818.74^{\mathrm{bc}}$ & $8665.05^{\mathrm{cde}}$ & $9278.51^{\mathrm{cd}}$ & $8610.78^{c}$ & $8533.42^{c}$ \\
\hline & June & $54.17^{\text {cde }}$ & $1480.81^{\mathrm{c}}$ & $4346.88^{\mathrm{cd}}$ & $7764.65^{\text {cdef }}$ & $9518.80^{\mathrm{cd}}$ & $9019.19^{c}$ & $8944.79^{c}$ \\
\hline & July & $29.56^{\mathrm{e}}$ & $878.29^{d}$ & $4060.59^{d}$ & $7031.17^{\text {ef }}$ & $9750.34^{\mathrm{cd}}$ & $9379.93^{c}$ & $9299.77^{c}$ \\
\hline & August & $31.41^{\mathrm{e}}$ & $504.25^{\mathrm{d}}$ & $2281.33^{\text {ef }}$ & $5667.85^{\mathrm{f}}$ & $6305.64^{\mathrm{e}}$ & $5411.75^{\mathrm{d}}$ & $5345.99^{d}$ \\
\hline & Mean & 71.01 & 1336.02 & 4707.40 & 7828.11 & 9253.17 & 8620.79 & 8415.20 \\
\hline \multirow[t]{10}{*}{ Gero Badeggi } & April & $196.07^{\mathrm{a}}$ & $2416.77^{a}$ & $9880.05^{\mathrm{a}}$ & $14671.47^{\mathrm{a}}$ & $15075.64^{\mathrm{a}}$ & $13878.40^{\mathrm{a}}$ & $13060.09^{\mathrm{a}}$ \\
\hline & May & $60.67^{\mathrm{cd}}$ & $1624.15^{\mathrm{bc}}$ & $5800.60^{\mathrm{bc}}$ & $11649.47^{\mathrm{b}}$ & $13170.00^{\mathrm{ab}}$ & $12493.27^{\mathrm{ab}}$ & $12413.64^{\mathrm{a}}$ \\
\hline & June & $41.56^{\mathrm{de}}$ & $1924.84^{\mathrm{bc}}$ & $3780.60^{\text {de }}$ & $10421.17^{\mathrm{bc}}$ & $12705.31^{\mathrm{ab}}$ & $11829.98^{\mathrm{ab}}$ & $11752.55^{\mathrm{ab}}$ \\
\hline & July & $30.18^{e}$ & $904.63^{\mathrm{d}}$ & $3028.64^{\text {def }}$ & $7593.74^{\text {def }}$ & $9183.83^{\mathrm{cd}}$ & $8795.51^{\mathrm{c}}$ & $8742.33^{c}$ \\
\hline & August & $26.18^{e}$ & $549.24^{\mathrm{d}}$ & $2088.09^{f}$ & $7240.11^{\text {ef }}$ & $8247.65^{\text {de }}$ & $6248.85^{\mathrm{d}}$ & $6187.02^{\mathrm{d}}$ \\
\hline & Mean & 70.90 & 1484.93 & 4915.60 & 10315.19 & 11676.44 & 10649.20 & 10431.13 \\
\hline & SL & & & & & & & \\
\hline & Planting date & * & * & * & * & * & * & * \\
\hline & Variety & ns & ns & ns & * & * & * & * \\
\hline & $\begin{array}{l}\text { Interaction } \\
(\mathrm{V} \times \mathrm{D})\end{array}$ & * & * & * & * & * & * & * \\
\hline
\end{tabular}

Values followed by the same superscript(s) indicated in columns are not significantly different at the $5 \%$ probability level when using Duncan multiple range test. SL- level of significance; ${ }^{*}$ significant at the 0.05 probability level; ns: values not significant; V-variety; D- planting date

Table 7: Effect of planting date on the number of tillers/plant of two varieties of pearl millet at Ekpoma

\begin{tabular}{|c|c|c|c|c|c|c|c|c|}
\hline \multirow[b]{2}{*}{ Crop variety } & \multirow[b]{2}{*}{ Planting date } & \multicolumn{7}{|c|}{ Weeks after planting } \\
\hline & & 2 & 4 & 6 & 8 & 10 & 12 & 14 \\
\hline \multirow[t]{6}{*}{ Gero Bida } & April & $1.00^{\mathrm{ns}}$ & $4.61^{\mathrm{a}}$ & $5.32^{\mathrm{a}}$ & $5.42^{\mathrm{a}}$ & $3.75^{\mathrm{a}}$ & $3.75^{\mathrm{ab}}$ & $3.25^{\mathrm{ab}}$ \\
\hline & May & 0.00 & $2.50^{c}$ & $3.25^{\mathrm{b}}$ & $3.27^{\mathrm{bc}}$ & $4.00^{\mathrm{a}}$ & $3.00^{\mathrm{bc}}$ & $2.58^{\mathrm{bcd}}$ \\
\hline & June & 0.00 & $1.97^{\mathrm{cd}}$ & $2.33^{c}$ & $2.92^{c}$ & $3.08^{\mathrm{ab}}$ & $2.33^{\mathrm{cd}}$ & $1.83^{\mathrm{de}}$ \\
\hline & July & 0.00 & $1.31^{\mathrm{de}}$ & $2.92^{\mathrm{bc}}$ & $3.08^{\mathrm{bc}}$ & $3.17^{\mathrm{ab}}$ & $2.42^{\mathrm{cd}}$ & $1.92^{\text {cde }}$ \\
\hline & August & 0.00 & $1.17^{\mathrm{e}}$ & $1.17^{\mathrm{d}}$ & $1.75^{\mathrm{d}}$ & $2.25^{\mathrm{b}}$ & $1.56^{\mathrm{d}}$ & $1.08^{\mathrm{e}}$ \\
\hline & Mean & 1.00 & 2.32 & 3.01 & 3.29 & 3.25 & 2.61 & 2.13 \\
\hline \multirow[t]{10}{*}{ Gero Badeggi } & April & 1.11 & $5.17^{\mathrm{a}}$ & $5.33^{\mathrm{a}}$ & $5.33^{\mathrm{a}}$ & $4.50^{\mathrm{a}}$ & $4.42^{\mathrm{a}}$ & $3.83^{\mathrm{a}}$ \\
\hline & May & 0.00 & $3.25^{\mathrm{b}}$ & $3.58^{\mathrm{b}}$ & $3.59^{\mathrm{bc}}$ & $3.59^{\mathrm{ab}}$ & $3.50^{\mathrm{ab}}$ & $3.00^{\mathrm{ab}}$ \\
\hline & June & 0.00 & $2.67^{\mathrm{bc}}$ & $3.17^{\mathrm{bc}}$ & $3.33^{\mathrm{bc}}$ & $3.33^{\mathrm{ab}}$ & $3.00^{\mathrm{bc}}$ & $2.58^{\mathrm{bcd}}$ \\
\hline & July & 0.00 & $1.67^{\mathrm{de}}$ & $3.67^{b}$ & $3.67^{b c}$ & $3.33^{\mathrm{ab}}$ & $3.33^{\mathrm{bc}}$ & $2.83^{\mathrm{bc}}$ \\
\hline & August & 0.00 & $1.17^{\mathrm{e}}$ & $2.33^{\mathrm{c}}$ & $3.92^{\mathrm{b}}$ & $4.33^{\mathrm{a}}$ & $3.75^{\mathrm{ab}}$ & $3.17^{\mathrm{ab}}$ \\
\hline & Mean & 1.11 & 2.78 & 3.62 & 3.97 & 3.82 & 3.60 & 3.08 \\
\hline & SL & & & & & & & \\
\hline & Planting date & ns & * & * & * & ns & * & * \\
\hline & Variety & ns & ns & * & * & ns & * & * \\
\hline & $\begin{array}{l}\text { Interaction } \\
(\mathrm{V} \times \mathrm{D})\end{array}$ & ns & * & * & * & * & * & * \\
\hline
\end{tabular}

Values followed by the same superscript(s) indicated in columns are not significantly different at the $5 \%$ probability level when using Duncan multiple range test. SL- level of significance; ${ }^{*}$ significant at the 0.05 probability level; ns: values not significant; V-variety; D- planting date. 
Table 8: Effect of planting date on stem girth $(\mathrm{cm})$ of two varieties of pearl millet at Ekpoma

\begin{tabular}{|c|c|c|c|c|c|c|c|c|}
\hline \multirow[b]{2}{*}{ Crop variety } & \multirow[b]{2}{*}{ Planting date } & \multicolumn{7}{|c|}{ Weeks after planting } \\
\hline & & 2 & 4 & 6 & 8 & 10 & 12 & 14 \\
\hline \multirow[t]{6}{*}{ Gero Bida } & April & $0.57^{\mathrm{a}}$ & $1.65^{\mathrm{a}}$ & $1.77^{\mathrm{abc}}$ & $2.08^{\mathrm{bc}}$ & $2.38^{\mathrm{a}}$ & $2.26^{\mathrm{bc}}$ & $2.19^{\mathrm{b}}$ \\
\hline & May & $0.41^{\mathrm{cd}}$ & $1.20^{\mathrm{bc}}$ & $1.83^{\mathrm{abc}}$ & $2.26^{\mathrm{ab}}$ & $2.35^{\mathrm{a}}$ & $2.38^{\mathrm{ab}}$ & $2.11^{b c}$ \\
\hline & June & $0.33^{\mathrm{de}}$ & $1.07^{\mathrm{cd}}$ & $1.63^{\mathrm{bc}}$ & $1.92^{\mathrm{cd}}$ & $2.08^{\mathrm{bc}}$ & $2.12^{\text {bcde }}$ & $2.07^{b c}$ \\
\hline & July & $0.27^{\mathrm{e}}$ & $0.91^{\mathrm{c}}$ & $1.53^{\mathrm{bc}}$ & $1.79^{\mathrm{d}}$ & $1.86^{\mathrm{bc}}$ & $1.82^{\mathrm{e}}$ & $1.75^{\mathrm{d}}$ \\
\hline & August & $0.26^{\mathrm{e}}$ & $0.87^{\mathrm{cd}}$ & $1.51^{\mathrm{bc}}$ & $1.73^{\mathrm{d}}$ & $1.74^{\mathrm{bc}}$ & $1.92^{\mathrm{de}}$ & $1.68^{d}$ \\
\hline & Mean & 0.37 & 1.14 & 1.66 & 1.96 & 2.08 & 2.10 & 1.96 \\
\hline \multirow[t]{10}{*}{ Gero Badeggi } & April & $0.55^{\mathrm{ab}}$ & $1.62^{\mathrm{ab}}$ & $2.11^{\mathrm{ab}}$ & $2.36^{\mathrm{a}}$ & $2.56^{\mathrm{a}}$ & $2.61^{\mathrm{a}}$ & $2.54^{\mathrm{a}}$ \\
\hline & May & $0.47^{\mathrm{bc}}$ & $1.53^{\mathrm{ab}}$ & $1.85^{\mathrm{ab}}$ & $2.22^{\mathrm{ab}}$ & $2.35^{\mathrm{a}}$ & $2.19^{\mathrm{bcd}}$ & $2.13^{b c}$ \\
\hline & June & $0.33^{\mathrm{de}}$ & $1.16 c^{d}$ & $1.81^{\mathrm{abc}}$ & $2.12^{\mathrm{abc}}$ & $2.15^{\mathrm{abc}}$ & $2.08^{\text {bcde }}$ & $2.02^{b c}$ \\
\hline & July & $0.26^{\mathrm{e}}$ & $0.82^{\mathrm{d}}$ & $1.55^{\mathrm{bc}}$ & $1.90^{\mathrm{cd}}$ & $1.92^{\mathrm{bc}}$ & $1.94^{\text {cde }}$ & $1.86^{\mathrm{cd}}$ \\
\hline & August & $0.26^{\mathrm{e}}$ & $0.84^{\mathrm{cd}}$ & $1.48^{c}$ & $1.94^{\mathrm{cd}}$ & $2.08^{\mathrm{abc}}$ & $2.08^{\text {bcde }}$ & $2.02^{b c}$ \\
\hline & Mean & 0.38 & 1.19 & 1.76 & 2.11 & 2.21 & 2.18 & 2.15 \\
\hline & SL & & & & & & & \\
\hline & Planting date & * & * & * & * & * & * & * \\
\hline & Variety & ns & ns & $\mathrm{ns}$ & * & * & * & * \\
\hline & $\begin{array}{l}\text { Interaction } \\
(\mathrm{V} \times \mathrm{D})\end{array}$ & * & * & * & * & * & * & * \\
\hline
\end{tabular}

Values followed by the same superscript(s) indicated in columns are not significantly different at the $5 \%$ probability level when using Duncan multiple range test. SL- level of significance; ${ }^{*}$ significant at the 0.05 probability level; ns: values not significant; V-variety; D- planting date.

number of days (Table 9). The mean number of days to flowering ranged from 54.67-68.67 days in 'Gero Bida' and 60.33-73.00 days in 'Gero Badeggi.' 'Gero Bida' flowered earlier than 'Gero Badeggi.' 'Gero Bida' needed an average of 62.93 days to flower while that of 'Gero Badeggi' was 68.40 days.

\subsection{DAYS TO $50 \%$ MATURITY}

Days to $50 \%$ maturity varied from $82.00-90.33$ days in 'Gero Bida' and 86.67- 96.33 days in 'Gero Badeggi' (Table 9). 'Gero Bida' matured earlier with a mean of 83.53 days than 'Gero Badeggi' with an average of 90.33 days. June plantings needed higher number of days to maturity while those of May needed lower number of days.

\subsection{LENGTH OF TERMINAL PANICLE PER PLANT}

The length of terminal panicle ranged from 41.88$54.75 \mathrm{~cm}$ and $55.17-81.58 \mathrm{~cm}$ in 'Gero Bida' and 'Gero Badeggi', respectively (Table 9). 'Gero Badeggi' panicles $(66.80 \mathrm{~cm})$ were longer than those of 'Gero Bida' $(44.46 \mathrm{~cm})$. However, April plantings had the longest panicle while July had the shortest.

\subsection{NUMBER OF TERMINAL PANICLES PER PLANT}

The result revealed that 'Gero Bida' and 'Gero Badeggi' sown at the five dates recorded 1.00 terminal panicle/ plant and did not differ significantly from each other (Table 9).

\subsection{NUMBER OF TILLER PANICLES PER PLANT}

Number of tiller panicles varied from 1.50 to 3.50 in 'Gero Bida' and 1.28-4.33 in 'Gero Badeggi' (Table 9). Although, 'Gero Badeggi' had higher number of tiller panicles/plant than 'Gero Bida', both were similar approximately 2 panicles per plant. April plantings had the highest number of tiller panicles/plant which differs significantly from other sown dates, while May plantings had the least.

\subsection{NUMBER OF SYNCHRONOUS TILLER (BRANCH) PANICLES PER PLANT}

The value for number branch panicles/plant ranged from 1.25 - 2.83 in 'Gero Bida' and 1.44 - 2.00 in 
Table 9: Effect of planting date on flowering and maturity and some yield components of two varieties of pearl millet

\begin{tabular}{|c|c|c|c|c|c|c|c|c|}
\hline Crop variety & Planting date & $\begin{array}{l}\text { Days to } 50 \% \\
\text { flowering }\end{array}$ & $\begin{array}{l}\text { Days to } 50 \% \\
\text { maturity }\end{array}$ & $\begin{array}{l}\text { Length of } \\
\text { terminal } \\
\text { Panicle/plant } \\
(\mathrm{cm})\end{array}$ & $\begin{array}{l}\text { Number of } \\
\text { terminal pani- } \\
\text { cle/plant }\end{array}$ & $\begin{array}{l}\text { Number of } \\
\text {-tiller panicle/ } \\
\text { plant }\end{array}$ & $\begin{array}{l}\text { Number of } \\
\text { synchronous } \\
\text { (branch) } \\
\text { panicle/plant }\end{array}$ & $\begin{array}{l}\text { Number } \\
\text { of seeds/ } \\
\text { panicle }\end{array}$ \\
\hline \multirow{6}{*}{ Gero Bida } & April & $68.67^{b c}$ & $82.67^{d}$ & $44.00^{\mathrm{e}}$ & $1.00^{\mathrm{ns}}$ & $3.50^{\mathrm{a}}$ & $2.67^{\mathrm{ns}}$ & $8439.00^{\mathrm{de}}$ \\
\hline & May & $54.67^{\mathrm{g}}$ & $79.00^{\mathrm{e}}$ & $54.75^{\mathrm{d}}$ & 1.00 & $1.53^{\mathrm{b}}$ & 2.56 & $10623.33^{b c}$ \\
\hline & June & $63.33^{\mathrm{e}}$ & $90.33^{\mathrm{b}}$ & $38.33^{\mathrm{e}}$ & 1.00 & $2.25^{\mathrm{b}}$ & 2.83 & $7437.00^{\mathrm{e}}$ \\
\hline & July & $66.33^{d}$ & $83.67^{d}$ & $41.88^{\mathrm{e}}$ & 1.00 & $1.75^{\mathrm{b}}$ & 1.25 & $8124.41^{\mathrm{de}}$ \\
\hline & August & $61.67^{\mathrm{ef}}$ & $82.00^{\mathrm{d}}$ & $43.33^{\mathrm{e}}$ & 1.00 & $1.50^{\mathrm{b}}$ & 2.17 & $8406.67^{\text {de }}$ \\
\hline & Mean & 62.93 & 83.53 & 44.46 & 1.00 & 2.11 & 2.29 & 8606.08 \\
\hline \multirow[t]{10}{*}{ Gero Badeggi } & April & $73.00^{\mathrm{a}}$ & $89.67^{b}$ & $81.58^{\mathrm{a}}$ & 1.00 & $4.33^{\mathrm{a}}$ & 2.00 & $11342.33^{\mathrm{ab}}$ \\
\hline & May & $60.33^{f}$ & $88.67^{b}$ & $70.67^{\mathrm{b}}$ & 1.00 & $1.28^{\mathrm{b}}$ & 1.44 & $12301.33^{\mathrm{a}}$ \\
\hline & June & $67.33^{\mathrm{cd}}$ & $96.33^{\mathrm{a}}$ & $55.17^{\mathrm{d}}$ & 1.00 & $2.25^{\mathrm{b}}$ & 1.67 & $9571.00^{\mathrm{cd}}$ \\
\hline & July & $72.00^{\mathrm{a}}$ & $90.33^{\mathrm{b}}$ & $59.75^{\mathrm{cd}}$ & 1.00 & $1.33^{\mathrm{b}}$ & 1.67 & $10698.50^{\mathrm{bc}}$ \\
\hline & August & $69.33^{\mathrm{b}}$ & $86.67^{c}$ & $66.83^{b c}$ & 1.00 & $1.75^{\mathrm{b}}$ & 1.83 & $10441.00^{\mathrm{bc}}$ \\
\hline & Mean & 68.40 & 90.33 & 66.80 & 1.00 & 2.19 & 1.72 & 10870.83 \\
\hline & SL & & & & & & & \\
\hline & Planting date & * & * & * & ns & * & ns & * \\
\hline & Variety & * & * & * & ns & ns & ns & * \\
\hline & $\begin{array}{l}\text { Interaction } \\
(\mathrm{V} \times \mathrm{D})\end{array}$ & * & * & * & ns & * & ns & * \\
\hline
\end{tabular}

Values followed by the same superscript(s) indicated in columns are not significantly different at the $5 \%$ probability level when using Duncan multiple range test. SL- level of significance; ${ }^{*}$ significant at the 0.05 probability level; ns: values not significant; V-variety; D- planting date

'Gero Badeggi' (Table 9). Although ‘Gero Bida' had higher number of branch panicles, both varieties produced approximately 2 panicles per branch. Crops planted in April had the highest number of panicle/branch while those planted in July had the least.

\subsection{NUMBER OF SEEDS PER PANICLE}

Crops planted in May had the highest number of seeds per panicle while those of June had the least (Table 9). The value ranged significantly from 7437.0010623.33 and 9571.00-12301.33 in 'Gero Bida' and 'Gero Badeggi', respectively. 'Gero Badeggi' had a higher $(10,870.83)$ number of seeds per panicle than 'Gero Bida' (8606.08).

\subsection{MASS OF 1000 SEEDS}

The mean 1000 seed mass ranged from 7.43-11.40 $\mathrm{g}$ in 'Gero Bida' and 7.63-12.70 g in 'Gero Badeggi' (Table 10). Although, 'Gero Badeggi' produced higher (9.24 g) mass than 'Gero Bida' ( $8.99 \mathrm{~g})$, but there was no sig- nificant difference. May planted crops had the highest while those of June had the least.

\subsection{TERMINAL PANICLE MASS}

April sown crops had the highest terminal panicle mass while those of July had the least (Table 10). The mean varied from 2.55-4.95 $\mathrm{t} \mathrm{ha}^{-1}$ and 4.63$6.45 \mathrm{t} \mathrm{ha}^{-1}$ in 'Gero Bida' and 'Gero Badeggi', respectively. On the whole, 'GeroBbadeggi' had a higher terminal panicle weight than 'Gero Bida.' 'Gero Bida' had $3.86 \mathrm{t} \mathrm{ha}^{-1}$ terminal panicle mass while 'Gero Badeggi' had $5.53 \mathrm{tha}^{-1}$.

\subsection{HUSK MASS}

The mean husk mass ranged from $0.82-2.74 \mathrm{tha}^{-1}$ in' Gero Bida' and 2.43-3.38 t ha ${ }^{-1}$ in 'Gero Badeggi' (Table 10). Crops sown in April produced the highest while those of June had the least. In all, 'Gero Badeggi' had a higher husk weight than 'Gero Bida' and they were 
Table 10: Effect of planting date on the grain yield and yield components of two varieties of pearl millet at Ekpoma

\begin{tabular}{|c|c|c|c|c|c|c|c|c|}
\hline Crop variety & Planting date & $\begin{array}{l}\text { Mass of } 1000 \\
\text { seeds }(\mathrm{g})\end{array}$ & $\begin{array}{l}\text { Terminal pan- } \\
\text { icle weight }\end{array}$ & Husk mass & $\begin{array}{l}\text { Grain mass } \\
\text { from terminal } \\
\text { panicles }\end{array}$ & $\begin{array}{l}\text { Grain mass } \\
\text { from syn- } \\
\text { chronous } \\
\text { ltiller (branch) } \\
\text { panicles }\end{array}$ & $\begin{array}{l}\text { Grain mass } \\
\text { from tiller } \\
\text { panicles }\end{array}$ & $\begin{array}{l}\text { Total grain } \\
\text { mass }\end{array}$ \\
\hline \multirow[t]{6}{*}{ Gero Bida } & April & $9.25^{c}$ & $4.95^{\mathrm{b}}$ & $2.74^{\mathrm{ab}}$ & $2.21^{\mathrm{d}}$ & $1.45^{\mathrm{abc}}$ & $2.52^{\mathrm{bc}}$ & $6.18^{\text {bcde }}$ \\
\hline & May & $11.40^{\mathrm{b}}$ & $4.36^{\text {bcd }}$ & $1.45^{\mathrm{de}}$ & $2.92^{\mathrm{b}}$ & $1.68^{\mathrm{ab}}$ & $2.57^{b c}$ & $7.17^{\mathrm{bc}}$ \\
\hline & June & $7.43 e$ & $3.65^{\mathrm{d}}$ & $1.91^{\mathrm{cd}}$ & $1.74^{\mathrm{f}}$ & $1.11^{\mathrm{bcd}}$ & $1.71^{\mathrm{c}}$ & $4.56^{\mathrm{ef}}$ \\
\hline & July & $8.88^{\mathrm{cd}}$ & $2.55^{\mathrm{e}}$ & $0.82^{\mathrm{e}}$ & $1.73^{\mathrm{f}}$ & $0.44^{\mathrm{d}}$ & $2.10^{c}$ & $4.27^{\mathrm{f}}$ \\
\hline & August & $7.97^{\mathrm{cde}}$ & $3.78^{\mathrm{cd}}$ & $1.47^{\mathrm{de}}$ & $2.31^{\mathrm{cd}}$ & $1.51^{\mathrm{abc}}$ & $1.66^{c}$ & $5.47^{\text {cdef }}$ \\
\hline & Mean & 8.99 & 3.86 & 1.68 & 2.18 & 1.24 & 2.11 & 5.53 \\
\hline \multirow[t]{10}{*}{ Gero Badeggi } & April & $8.25^{\text {cde }}$ & $5.97^{\mathrm{a}}$ & $3.38^{\mathrm{bc}}$ & $2.59^{c}$ & $0.58^{\mathrm{d}}$ & $1.76^{c}$ & $4.93^{\mathrm{def}}$ \\
\hline & May & $12.70^{\mathrm{a}}$ & $5.82^{\mathrm{a}}$ & $2.45^{\mathrm{bc}}$ & $3.37^{\mathrm{a}}$ & $1.83^{\mathrm{a}}$ & $4.13^{\mathrm{a}}$ & $9.33^{\mathrm{a}}$ \\
\hline & June & $7.63^{\mathrm{de}}$ & $4.63^{\mathrm{bc}}$ & $2.43^{\mathrm{bc}}$ & $2.16^{\mathrm{de}}$ & $1.98^{\mathrm{a}}$ & $3.65^{\mathrm{ab}}$ & $7.79^{\mathrm{ab}}$ \\
\hline & July & $9.03^{c}$ & $4.76^{\mathrm{b}}$ & $2.88^{\mathrm{ab}}$ & $1.87^{\mathrm{ef}}$ & $0.46^{\mathrm{d}}$ & $2.20^{c}$ & $4.53^{\mathrm{ef}}$ \\
\hline & August & $8.57^{\mathrm{cde}}$ & $6.45^{\mathrm{a}}$ & $3.12^{\mathrm{ab}}$ & $3.33^{\mathrm{a}}$ & $0.87^{\mathrm{cd}}$ & $2.24^{\mathrm{c}}$ & $6.44^{b c d}$ \\
\hline & Mean & 9.24 & 5.53 & 2.85 & 2.66 & 1.14 & 2.80 & 6.60 \\
\hline & SL & & & & & & & \\
\hline & Planting date & * & * & * & * & * & * & * \\
\hline & Variety & ns & * & * & * & * & * & * \\
\hline & $\begin{array}{l}\text { Interaction } \\
(\mathrm{V} \times \mathrm{D})\end{array}$ & * & * & * & * & * & * & * \\
\hline
\end{tabular}

Values followed by the same superscript(s) indicated in columns are not significantly different at the $5 \%$ probability level when using Duncan multiple range test. SL- level of significance; ${ }^{*}$ significant at the 0.05 probability level; ns: values not significant; $\mathrm{V}$-variety; D- planting date

significantly different. The average of $2.85 \mathrm{t} \mathrm{ha}^{-1}$ was for 'Gero Badeggi' while that of 'Gero Bida' was $1.68 \mathrm{t} \mathrm{ha}^{-1}$.

\subsection{GRAIN MASS FROM TERMINAL PANICLE}

Crops planted in May had the highest grain mass from terminal panicles while those of July had the least (Table 10). Grain mass obtained from terminal panicles varied from 1.73- $2.92 \mathrm{t} \mathrm{ha}^{-1}$ and 1.87- 3.37 $\mathrm{t} \mathrm{ha}^{-1}$ in 'Gero Bida' and 'Gero Badeggi' respectively (Table 10). On the whole, 'Gero Badeggi' had heavier (2.67 $\mathrm{t} \mathrm{h}^{-1}$ ) grains from terminal panicle than 'Gero Bida' $(2.18 \mathrm{t}$ $\left.\mathrm{ha}^{-1}\right)$.

\subsection{GRAIN YIELD FROM SYNCHRONOUS TILLER (BRANCH) PANICLES}

The value ranged from $0.44-1.68 \mathrm{t} \mathrm{ha}^{-1}$ in 'Gero Bida' and 0.46-1.98 t ha ${ }^{-1}$ in 'Gero Badeggi' (Table 10). Although grain yield from branches was higher in 'Gero Bida' (1.24 tha ${ }^{-1}$ ) than 'Gero Badeggi' (1.14 $\left.\mathrm{t} \mathrm{ha}^{-1}\right)$, how- ever, there was no significant difference. Crops sown in May had the highest grain yield from synchronous tiller panicles (branch) while those sown in July had the least.

\subsection{GRAIN YIELD FROM TILLER PANICLES}

Grain yield from tillers varied from 1.66$2.57 \mathrm{t} \mathrm{ha}^{-1}$ and 1.76-4.13 $\mathrm{t} \mathrm{ha}^{-1}$ in 'Gero Bida' and 'Gero Badeggi' respectively (Table 10). In all, 'Gero Badeggi' had higher grain mass from tillers than 'Gero Bida'. The mean of $2.80 \mathrm{t} \mathrm{ha}^{-1}$ was for 'Gero Badeggi' while that of 'Gero Bida' was $2.11 \mathrm{t} \mathrm{ha}^{-1}$. However, the highest and the least grain yield from tiller panicles were obtained from crops planted in May and August, respectively.

\subsection{TOTAL GRAIN YIELD}

Total grain yield ranged from 4.27-7.17 $\mathrm{t} \mathrm{ha} \mathrm{h}^{-1}$ in 'Gero Bida' and 4.53-9.33 t ha ${ }^{-1}$ in 'Gero Badeggi' (Table 10). On the whole, 'Gero Badeggi' had significantly higher $\left(6.51 \mathrm{t} \mathrm{ha}^{-1}\right)$ total grain yield than 'Gero Bida' 
$\left(5.53 \mathrm{t} \mathrm{ha}^{-1}\right)$. The order of performance in total grain yield was May $>$ June $>$ August $>$ April $>$ July. However, May and July sown crops significantly gave the highest and the least total grain yield, respectively.

\section{DISCUSSION}

Nutrient status of soil is a paramount index in crop production and food security. The soil nutrients of the site were low when compared with the critical levels of $10 \mathrm{~g} \mathrm{~kg}^{-1}$ organic carbon, $15 \mathrm{~g} \mathrm{~kg}^{-1}$ total nitrogen and $15 \mathrm{mg} \mathrm{kg}^{-1}$ phosphorus (Kparmwang et al., 2001). However, keeping in view the low nutrient status of the soil, grain yields obtained from this study commensurate with those reported by Uzoma et al. (2010) from relatively higher nutrients based soils in the guinea savannah zone of Nigeria. Fahmy et al. (2010) reported that pearl millet can grow in a wide range of environments and soils.

Plant height is an important component in the determination of growth (Khan et al., 2009). Pearl millet sown in April significantly developed the tallest stems while those planted in August developed the shortest. The possible reason is that early sown crops had availed prolonged vegetative growth, as a result, plant attained maximum height as compared to late sown crops. These results are in line with those of Maas et al. (2007) who reported that crops sown on $15^{\text {th }}$ June produced significantly taller plants than those sown on $15^{\text {th }}$ July.

There were significant $(p<0.05)$ differences in number of leaves/plant among planting dates of pearl millet varieties throughout the period of the study, but effect of variety was not significant from 2-6 weeks after planting. The early sown crops produced more leaves than late planted crops due to the utilization of more assimilate in producing more leaves. These results are in conformity with the findings of Andrews et al. (1998); Amanullah et al. (2015) who attributed the higher number of leaves produced by early sown crops to available moisture which enhanced utilization of more assimilate.

Millet tillering was divided into sub-terminal tillers and synchronous tillers. Sub-terminal tillers are those which arise from auxiliary bud axis while the synchronous tillers are those from the base of the leaf bud (Obeng et al., 2012). The later was referred to as branches (Omoregie \& Nwajei, 2015). Number of branches/plant was significantly affected by planting date and the interaction between variety and planting date, but the effect of variety was not significant. Although 'Gero Bida' was higher than 'Gero Badeggi' with regards to number of branches, but there was no signifi- cant difference. Generally, time of planting significantly influenced the growth and development of synchronous tillers (branches) produced in both varieties. The reduction in the number of synchronous tillers after 12 WAP in both varieties was due to senescence. Obeng et al. (2012); Omoregie \& Nwajei (2015) observed significant decline in the number of synchronous tillers owing to senescence which agreed with the present study.

Total plant feeding area ensures adequate photosynthesis for optimum growth and development. The leaf area of pearl millet differed significantly $(\mathrm{p}<0.05)$ among sowing dates and planting $\times$ variety interaction, but variety was not significant from 2-6 WAP. Crops sown at early date (April/May) had the largest leaf area compared to the later dates due to moisture absorption ability and high conversion of assimilates. This is in agreement with the results of a study conducted elsewhere by Agber et al. (2012) who reported that the largest leaf area of pearl millet with early sown date was due to the availability of water during vegetative growth which increased the total leaf area of the crop.

Results in Table 6 revealed that number of tillers/ plant was significantly affected by time of planting except at 2 and 10 WAP. Number of tillers/ plant had significant difference for crops at 6, 812 and 14, while the interaction between plating date and variety were significant from 4 -14 WAP. This sub-terminal tillers were significantly higher when pearl millet was planted in April which reflects the crops ability to develop various vegetative and reproductive parts during early plantings with favourable environmental conditions. Uzoma et al. (2010) reported significant difference in number sub terminal tillers among sowing dates due to environmental responds.

Stem girth is a measure of plants resistance or susceptibility to lodging, but adequate stand establishment ensures good growth and stability of crops. Stem girth was significantly affected by planting date and planting date $\mathrm{x}$ variety interaction throughout the weeks after planting, but variety had no significant effect from 2 - 6 WAP. Early planted crops had the biggest girths and were significantly at par from late sown crops. This is consistent with the findings of Uzoma et al. (2010); Omoregie \& Nwajei (2015) who report that timeliness in planting is necessary as it has great effect on the growth of millet because it places the crops growth cycle within the distribution of rains which in turn enhance good plant architecture.

Planting date, variety and planting date $\times$ variety interaction significantly affected days to $50 \%$ flowering and maturity. However, crops sown in May needed lower number of days to flowering and maturity than other dates. This is in agreement with the earlier find- 
ings by Uzoma et al. (2010); Omoregie \& Nwajei (2015) who found significant difference in days to $50 \%$ flowering and maturity of pearl millet and crops that flowered early also matured early.

Results on yield and yield components of pearl millet showed that planting date, variety and planting date $\mathrm{x}$ variety interaction had significant effect on length of terminal panicle/plant, number of seeds/panicle, terminal panicle mass and husk mass. There was no significant difference among the varieties in number of tiller panicles and mass of 1000 seeds while number of terminal and synchronous tiller panicles were not significantly affected by planting date, variety and planting date $\mathrm{x}$ variety interaction. On the whole, 'Gero Badeggi' had higher yield components than 'Gero Bida' except number of synchronous tillers/ plant. Early sown crops significantly had higher yield components than late sown crops. These outstanding performances of pearl millet yield components by early sown crops over late planted crops were because early sown crops had well favourable weather conditions and gained prolonged growth period as a result more assimilates were accumulated for growth and development as well as grain production. The differences in varietal responds could have been as a result of difference in partitioning of assimilates to the grain and genotypic variation. Uzoma et al. (2010); Omoregie \& Nwajei (2015) observed significant difference in yield components of millet and reported that the variety with highest yield components gave the highest grain mass which was observed in this study.

The grain yield from terminal panicles, tiller panicles, synchronous tiller (branch) panicles and total grain yield were significantly affected by planting date, variety and planting date $\mathrm{x}$ variety interaction. Early sown crops gave heavier grains than late sown crops due to prolonged growth period with ideal growth conditions and better vegetative characters. This result is in agreement with the results reported by Tanzubi et al. (2002); Leila (2008) who observed that pearl millet planted in May had higher grain yield due to better vegetative growth, well distributed and favourable weather conditions during grain development. This yield reduction from crops planted in June - August were due to the plants' inability to attain optimum energy reserves. This is consistent with the report of Uzoma et al. (2010) that substantial yield loss may be associated with slight delay in planting as in the case those planted from June-August or slight earliness in planting as of those planted in April which agrees with our results.

\section{CONCLUSION}

This study concluded that planting date of pearl millet influenced the grain yield from synchronous till- er (branch) and tiller panicles as components of total grain yield with early sown crops being more favourable than later dates. Thus, pearl millet planted in April is regarded as the option for vegetative characters and ideal for pearl millet grown for forages while May is the option for the grain production in Ekpoma, Edo state, Nigeria.

\section{REFERENCES}

Agber, P.I., Ter, S., \& Ayuba, S.A. (2012). Assessment of pearl millet performance to early and late planting opportunities and fertilizer application models in Makurdi, Nigeria. African Journal of Agricultural Research, 7, 5955-5961. https://doi.org/10.5897/AJAR12.2051

Amanullah, J., Imran, K., Shahzad, A., \& Amir, S. (2015). Sowing date and sowing methods influence on growth, yield and yield components of pearl millet under rainfied conditions. Journal of Environmental and Earth Science, 5, 2224-3216.

Amodu, J.T., Adamu, A.M., Adeyinka, I.A., Alawa, J.P., \& Jegede, J.O. (2005). The nutritive value of laboratory ensiled lablab (Lablab purpureus) and pearl millet (Pennisetum americanum). Tropical GrassLand, 39, 117-123.

Anderson, J.M. \& Ingram, J.S. (1993). Tropical soil biology and fertility. A hand book of soil methods (pp. 23-45). Information and press Eynsham.

Andrews, D.J., Rajewski, J.F., \& Manson, S.C. (1998). Grain pearl millet: A newly developed crop. Extension Vision 2, 2-6.

Fahmy, A.A., Youssef, K.M., \& Elshaer, H.M. (2010). Intake and nutritive value of some salt tolerant fodder grasses for sheep under saline conditions of South Sinai, Egypt. Small Ruminant Research, 91, 110-115. https://doi.org/10.1016/j. smallrumres.2009.11.023

Gabatshele, M.L., Thembinkosi, M., Phizana, M., Christopher, M., \& Witness, M. (2014). The growth and development of two pearl millet landraces as affected by intra row spacing. International Journal of Current Microbiology and Applied Sciences, 3, 505-515.

Ighalo, S.O., \& Remison, S.U. (2010). Effect of weeding frequency and nitrogen application on the vegetative growth of Rice. Nigeria Journal of Agriculture and Forestry, 2, 219 $-232$.

Khan, A.Z., Shah, P., Khalil, S.K., \& Taj, F.H. (2003). Influence of planting date and plant density on morphological traits of determinate and indeterminate soybean cultivars under temperate environment. Pakistan Journal of Agronomy, 2, 146-152. https://doi.org/10.3923/ja.2003.146.152

Khan, Z.H., Khalil, S.K., Nigar, S., Khalil, I., Haq, I., Ahmad, I., Ali, A., \& Khan, M.V. (2009). Phenology and yield of sweet corn landraces influenced by planting dates. Sahad Journal of Agriculture, 25, 153-157.

Kparmwang, T., Chude, V.O. and Abdullahi, D. (2001). The classification and genesis of benchmark soils of Bauchi State, Nigeria. Journal of Soil Science, 14, 30-39.

Lawn, R.J., Summerfield, R.J., Ellis, R.H., Roberts, E.H., Chay, 
P.M., Brouwer, J.B., Rose, J.L. \& Yeates, S.J. (1993). Towards the reliable prediction of time to flowering in six annual crops. VI. Applications in crop improvement. Experimental Agriculture, 31, 89-108. https://doi.org/10.1017/ S0014479700025047

Leila, R. (2008). Autochthonous pearl millet ecotype (Pennisetum glacucum L. R. Br.) responds to different sowing dates in Tunisia. Sjemenarstre, 25,123-135.

Maas, A.L., \& Hanna, W.W. and Mullinix, B.G. (2007). Planting date and row spacing affecting grain yield and height of pearl millet tifgrain 102 in the South Eastern Coastal plain of the United States. Journal of Semi-Arid Tropics of Agricultural Research, 5, 1-4.

Obeng, E., Ernest, C., Bharat, P.S., Rafina, W., Leapold, M.N., \& David, M.A. (2012). Growth and grain yield of pearl millet genotypes at different levels of nitrogen fertilization in the south-eastern United State. Journal of Agricultural Science, 4, 155-160. https://doi.org/10.5539/jas.v4n12p155

Omoregie, A.U., \& Nwajei, S.E. (2015). Evaluation of some varieties of pearl millet (Pennisetum typhoides) in a forestsavanna transition zone of Edo State, Nigeria. In:Omeje, S.I., Emosairue, C.C., Chukwuji, C.C., Bratte, L., Isikwenu, J.O., Iaiorhovoja, R.A. and Agbogidi, M.O. (eds). Agriculture: The Nigerian economy beyond oil. Proceedings of the $49^{\text {th }}$ Annual Conference of the Agricultural Society of Nige- ria held at Delta State University, Asaba Campus, Asaba, Nigeria 9-13 ${ }^{\text {th }}$ November, 2015. 2, 1346-1349.

Raemaeker, R.H. (2001). Crop production in tropical Africa, Brussels, Belgium. pp. 47-57.

Remison, S.U., \& Eifediyi, E.K. (2014). Effects of different organic materials and NPK fertilizer on the performance of maize (Zea mays L.). Poljoprivreda, 20, 16-21.

Statitical Analysis System. (2002). SAS user's guide: Statistics version 9.0 Cary, NC, USA: SAS Institute Inc., 2002.

Shinggu, C.P. and Gani, M. (2012). Effects of planting methods, sowing dates and spacing on weed and the prductivity of finger millet (Eleusine corocana (L.) Gaertn) in the northern guinea-savanna of Nigeria. Global Journal of Bio-Science and Biotechnology, 1,160-162.

Tanzubil, P.B., Mensah, G.W.K. \& McCaffery, A.R. (2002). Effect of millet variety and date of sowing on diapauses development in Coniesta ignfusalis (Hampson) in Northern Ghana. Journal of Crop Protection, 21, 793-798. https://doi. org/10.1016/S0261-2194(02)00039-X

Uzoma, A.O., Eze, P.C., Alabi, M., Mgbonu, F., Aboje, J.E. \& Osunde, A.O. (2010). Effects of variety and planting date on the growth and yield of pearl millet in the southern guinea- savanna zone of Nigeria. Journal of Agriculture and Veterinary Science, 2, 122-126 